\title{
Molecular imaging for stem cell therapy in the brain
}

\author{
Nora Sandu ${ }^{1,2}$, Tumul Chowdhury ${ }^{3}$ and Bernhard Schaller ${ }^{1,2^{*}}$ \\ See related research by Micci et al., http://www.stemcellres.com/content/6/1/131
}

\begin{abstract}
Molecular imaging is one of the methods to follow-up stem cell therapy by visualization in the brain. In a recent article in Stem Cell Research \& Therapy, Micci et al. offer a thorough discussion of the advantages and disadvantages of this method and their roles in the future. The authors are among the very first who have implemented recently introduced molecular imaging techniques in experimental research and clinical practice.
\end{abstract}

\section{Background}

Current neuroimaging techniques give very limited insight into molecular and cellular sequences of events. Therefore, the exact underlying mechanism by which neural stem cells target physiological or pathological brain areas remains elusive. Monitoring of these cells is currently also carried out by the use of various modes of molecular imaging, which is a (novel) technology for visualizing metabolism and signal transduction to gene expression. Most importantly, molecular imaging will render possible the identification of potential therapeutic targets in the development of new treatment strategies and in their successful implementation in clinical application.

\section{Discussion}

In such a context, Micci et al. [1] have demonstrated the human sodium iodide symporter (hNIS), a transmembrane glycoprotein and widely used probe, as a new transporter-based reporter gene for non-invasive molecular imaging and point out one of the principal challenges of molecular imaging of the brain [2-6]. Such new reporter genes will help find the best target

\footnotetext{
* Correspondence: bernhardjschaller@gmail.com

'University of Oxford, Wellington Square, Oxford OX1 2JD, UK

${ }^{2}$ University of Southampton, University Road, Southampton SO17 1BJ, UK

Full list of author information is available at the end of the article
}

on a neurochemical as well as on a commercial level as applying stem cell transplantation in a clinical medium, which is necessity for analysis of the benefit to detect, localize, and examine the stem cells in vivo at both cellular and molecular levels.

Molecular imaging of stem cells has two principal advantages over other methods [6-11]: it allows visual representation, characterization, and quantification of biological processes in the same live recipient over time and it is non-invasive. In this context, hNIS may be an alternative to already-existing vectors like HSV-1 TK or D2R. As hNIS neither is physiologically expressed in the brain nor crosses the intact blood-brain barrier, we can directly examine how it relates to enzymatic activity or gene expression, being perhaps essential to obtain a more detailed description of the failure or success of the cell therapy. Therefore, hNIS incorporated into vectors will make it possible to more fully understand with a relatively high sensitivity the function of protein networks and their role in the spread of stem cells. Such knowledge will facilitate the discovery of still more informative biomarkers.

This restriction leads us to the question of which vector probes should be ideal for molecular imaging of cell therapy. Unfortunately, we think that it is easier to answer this question in different ways. A new vector probe should be able to help to answer some of the ongoing questions in stem cell therapy: the most efficacious route of delivery, the appropriate choice of stem cell type(s), the optimal cell population for treatment in a chronic setting, and the favorable time-point of cell delivery. A safe, non-invasive, and repeatable imaging modality that could identify injected stem cells would be able to answer questions about cell viability and retention as well as provide the ability to adjust the assessment of bioactivity on the basis of actual delivered doses of cells [7, 8]. In the long term, stem cell-derived regeneration still faces difficulties in its efforts to improve because of the need to monitor stem cells continuously with high 
temporal resolution and good biocompatibility. Under such circumstances, the properties of differentiation and self-renewal of stem cells over long periods of time might be of importance. Moreover, multimodality imaging reporter genes will allow us to choose the imaging technologies that are most appropriate for the biologic problem at hand and facilitate the clinical application of reporter gene technologies.

The investigation by Micci et al. [1] is in line with these current questions of stem cell research, but we have to be aware that stem cell therapy can be used for the clinical daily practice only if safety and efficacy of the transplanted cells can be guaranteed. However, inefficient stem cell differentiation, difficulty in verifying successful delivery to the target organ, and problems with engraftment all hamper the transition from laboratory animal studies to human clinical trials [7-9]. Therefore, there is a need to refine and optimize tracking techniques, as is done by Micci et al. [1]. Moreover, instrumentational improvements, the identification of novel targets and genes, and imaging probe developments suggest that moleculargenetic imaging is likely to play an increasingly important role in the diagnosis and therapy of some brain diseases [11-14]. Fortunately, the advent of molecular imaging will continue to lead unprecedented progress in understanding the fundamental behavior of stem cells, including their survival, bio-distribution, immunogenicity, and tumorogenicity, in the targeted tissues of interest.

\section{Conclusions}

Molecular imaging opens a new door to stem cell therapy, not only in treatment monitoring but also in better understanding the underlying (molecular) processes. Tracking techniques are an important part of this imaging progress and underline the important impact of molecular imaging on patient management with stem cell therapy of the brain.

\footnotetext{
Abbreviation

hNIS: human sodium iodide symporter.
}

\section{Competing interests}

The authors declare that they have no competing interests.

\section{Authors' contributions}

NS, TC, and BS drafted the manuscript. All authors read and approved the final manuscript.

\section{Author details}

${ }^{1}$ University of Oxford, Wellington Square, Oxford OX1 2JD, UK. ${ }^{2}$ University of Southampton, University Road, Southampton SO17 1BJ, UK. ${ }^{3}$ Department of Anesthesiology and Peri-operative Medicine, University of Manitoba,

Winnipeg, MB R3T 2N2, Canada.

Published online: 18 December 2015

\section{References}

1. Micci M-A, Boone DR, Parsley MA, Wei J, Patrikeev I, Motamedi M, et al. Development of a novel imaging system for cell therapy in the brain. Stem Cell Res Ther. 2015;6:131.
2. Spiriev T, Sandu N, Schaller B. Molecular imaging and tracking stem cells in neurosciences. Methods Mol Biol. 2013;1052:195-201.

3. Sandu N, Spiriev T, Schaller B. Stem cell transplantation in neuroscience: the role of molecular imaging. Stem Cell Rev. 2012;8:1265-6.

4. Sandu N, Schaller B. Molecular imaging of stem cell therapy in brain tumors: a step towards personalized medicine. Arch Med Sci. 2012:8:601-5.

5. Sandu N, Momen-Heravi F, Sadr-Eshkevari P, Schaller B. Molecular imaging for stem cell transplantation in neuroregenerative medicine. Neurodegener Dis. 2012;9:60-7.

6. Sandu N, Pöpperl G, Toubert ME, Spiriev T, Arasho B, Orabi M, et al. Current molecular imaging of spinal tumors in clinical practice. Mol Med. 2011;17:308-16.

7. Sandu N, Schaller B. Stem cell transplantation in brain tumors: a new field for molecular imaging? Mol Med. 2010;16:433-7.

8. Schaller BJ, Cornelius JF, Sandu N, Buchfelder M. Molecular imaging of brain tumors personal experience and review of the literature. Curr Mol Med. 2008:8:711-26.

9. Schaller B, Cornelius JF, Sandu N. Molecular medicine successes in neuroscience. Mol Med. 2008;14:361-64.

10. Schaller BJ. Strategies for molecular imaging dementia and neurodegenerative diseases. Neuropsychiatr Dis Treat. 2008;4:585-612.

11. Schaller BJ, Modo M, Buchfelder M. Molecular imaging of brain tumors: a bridge between clinical and molecular medicine? Mol Imaging Biol. 2007;9:60-71.

12. Sandu N, Momen-Heravi F, Sadr-Eshkevari P, Arvantaj A, Schaller B. Molecular imaging of stem cells: a new area for neuroscience. In: Schaller B, editor. Molecular imaging. Rijeka, Croatia: InTech; 2012. p. 211-20.

13. Sandu N, Pöpperl G, Toubert ME, Arasho B, Spiriev T, Orabi M, et al. Molecular imaging of potential bone metastasis from differentiated thyroid cancer: a case report. J Med Case Rep. 2011;5:522.

14. Schaller B. Influences of brain tumor-associated $\mathrm{pH}$ changes and hypoxia on epileptogenesis. Acta Neurol Scand. 2005;111:75-83. 